\title{
Using blocks for constraint satisfaction
}

\section{Report}

Author(s):

Seybold, Bernhard; Metzger, Felix; Ogan, Gül; Simon, Klaus

Publication date:

1998

Permanent link:

https://doi.org/10.3929/ethz-a-006652838

Rights / license:

In Copyright - Non-Commercial Use Permitted

Originally published in:

Technical report 297 


\title{
Using Blocks for Constraint Satisfaction
}

\author{
B. Seybold * \\ F. Metzger \\ G. Ogan \\ K. Simon
}

\begin{abstract}
The influence of graph-related properties on constraint satisfaction problems (CSP) has been investigated thoroughly. In this paper, we analyze the impact of a further graph concept on CSPs: biconnected components (blocks). They are an important structure which we exploit for constraint satisfaction in various ways. First, we show that a weaker kind of consistency- $k$-block-consistency ( $k$-BC) - is sufficient for $k$-consistency under a certain condition on the used variable ordering which we call prefix-connected. $\mathrm{BC}$ can be computed more efficiently than other consistencies, because it is less restrictive. Second, we show that redundancy and inconsistency can be detected on the block level. This remains invariant during $\mathrm{BC}$ enforcement, since $\mathrm{BC}$ enforcement preserves the block structure. Third, we show that the width $k$ of a graph is bounded by $k_{\max } \leq k \leq k_{\max }+1$ where $k_{\max }$ is the maximum width of the block induced subgraphs.
\end{abstract}

\section{Introduction}

In general, a CSP can be attacked by two strategies: consistency enforcement or search. Algorithms work best if they combine these strategies in a clever way. However, they still need exponential time in the worst case. But, graph theoretic properties can be used to define tractable subclasses.

One of the most important concepts is the width introduced by FrEUDER [Fre82]. He showed that a solution to a CSP can be found in time polynomial in the width. Later, he extended this concept to $j$-width, showing that the problem can be solved in time exponential in $j+1$, where $j$ is the smallest number such that the $j$-width of the graph is 1 . In this context, he also shows that a graph has a $(b-1)$-width of 1 where $b$ is the size of the largest block. This makes implicitly use of blocks. However, he does not fully exploit the properties of blocks, e.g. that a weaker kind of consistency is sufficient for consistency or that blocks bound redundancy.

Efficiency can be increased by observing that consistency need not be enforced on each tuple if the variable ordering is known in advance and fixed during search. Directed consistency [DP88] applies consistency such that only support in variables which are later instantiated is required. Adaptive consistency [DP88] belongs to the same category and makes use of the observation that consistency must only be applied in the parents of each vertex w.r.t. a given ordering. Blocks also take the neighborhood into account, but contrary to adaptive consistency this can be done a priori and more explicitly.

In this paper, we analyze how blocks, which have been well-known in graph theory for over twenty years ([Tar72]), influence constraint satisfaction problems. Based on the block structure, we define a weaker consistency-block-consistency $(\mathrm{BC}) . \mathrm{BC}$ restricts less variable tuples than other kinds of consistency. BC enforcement has the nice property that it preserves the block structure, such that block-based observations remain invariant. We will show that $\mathrm{BC}$ is sufficient for directed consistency if the ordering is prefix-connected. Blocks give us also the opportunity to analyze the complexity of a CSP instance more precisely, which has to a certain extent already been noticed by FreUder [Fre85].

\footnotetext{
*\{seybold, simon\}@inf.ethz.ch, \{metzger, ogan\}@iwf.bepr.ethz.ch

Research supported in part by Swiss Government, KTI 2726.1.
} 
In addition, blocks determine how redundancy and inconsistency are bounded in the network. It can be shown that the existence of at least one additional path between two adjacent vertices is necessary for both properties. This is reflected by the block structure. This observation is an invariant during $\mathrm{BC}$ enforcement. The block structure can be calculated efficiently (linear in the size of the network) and the methods presented in this paper are orthogonal to other solving schemes. Therefore they may be cheaply introduced into existing search techniques.

The width of the graph and the width of the block induced subgraph are connected. We show that the width $k$ of a graph is bounded by $k_{\max } \leq k \leq k_{\max }+1$ where $k_{\max }$ is the maximum width of the block induced subgraphs. The width $k$ is also bounded to be $\leq b$ where $b$ is the maximum of 2 and the size of the largest block. This can be used to show that there is a backtrack-free search order if the graph is strongly directed $b$-block consistent.

The paper is organized as follows. In Section 2 basic definitions are given. In Section 3 we introduce block consistency and show how it can be exploited for solving algorithms. How blocks influence redundancy and inconsistency spreading is analyzed in Section 4. In Section 5 the width of the entire graph and those of the blocks are linked with each other.

\section{Definition and Notation}

A constraint satisfaction problem (CSP) is described by an undirected graph $G=(V, E)$ where the vertices represent the variables of the CSP and the edges represent the (binary ${ }^{1}$ ) non-trivial constraints. Each variable $i$ has a domain $D_{i}$ which represents the set of all possible values of $i$. A constraint $C_{i j}$ is a subset of $D_{i} \times D_{j}$. It is neither empty (always inconsistent) nor complete (no edge). We will use the terms "vertex" and "edge" whenever we treat graph related topics, and "variable" and "constraint" for CSP topics.

Two vertices are adjacent if they are connected by an edge. The adjacency of a vertex $v$, $\operatorname{adj}(v)$, is the set of all adjacent vertices of $v$. The degree of a vertex is the size of the adjacency. A path from $v$ to $w$ is a sequence of vertices such that each two consecutive vertices are adjacent. Two paths are vertex disjoint if they do not share any vertices except the start or end vertex. Two vertices are biconnected if there are at least two vertex disjoint paths between them. A block $B$ (biconnected component) is a maximal subset of vertices where each two nodes are biconnected, $|B|$ the size of $B$. If there is a triple of distinct vertices $a, b$, and $v$, such that each path from $a$ to $b$ goes through $v$ and there exists at least one such path, then $v$ is called an articulation point. A bridge is an edge connecting vertices which are not in the same block. Two blocks $B_{1}$ and $B_{2}$ are adjacent if they have a vertex in common or if they are connected by a bridge. The block graph $G^{\prime}=\left(V^{\prime}, E^{\prime}\right)$ of a graph $G=(V, E)$, given $V^{\prime}$ the blocks of $G$, is constructed as follows [Fre85]. The connectivity components are treated separately. Choose one of the blocks to be the root of the tree and put it into a queue $Q$. While $Q$ is not empty, remove a block $b$ out of the queue. Then put all not yet considered adjacent blocks ${ }^{2}$ into $Q$ and add an edge between $b$ and each of them. The block graph $G^{\prime}$ of a graph $G$ is a tree iff $G$ is connected and a forest iff $G$ is not connected. The block graph $G^{\prime}$ of a graph $G$ is a spanning forest for the graph where the vertices are the blocks of $G$ and there is an edge if two blocks are adjacent.

A tuple of values for a tuple of variables is allowed if it satisfies all constraints among the variables. A support is a value for a certain variable which extends an allowed value tuple to a bigger allowed tuple. A CSP is $k$-consistent if each allowed value tuple for $k-1$ variables has

\footnotetext{
${ }^{1}$ In this paper, we restrict ourselves to the binary case. Most results, however, can easily be adapted to the $n$-ary case.

${ }^{2}$ FREUDER only considers blocks which share an articulation point and misses bridges.
} 


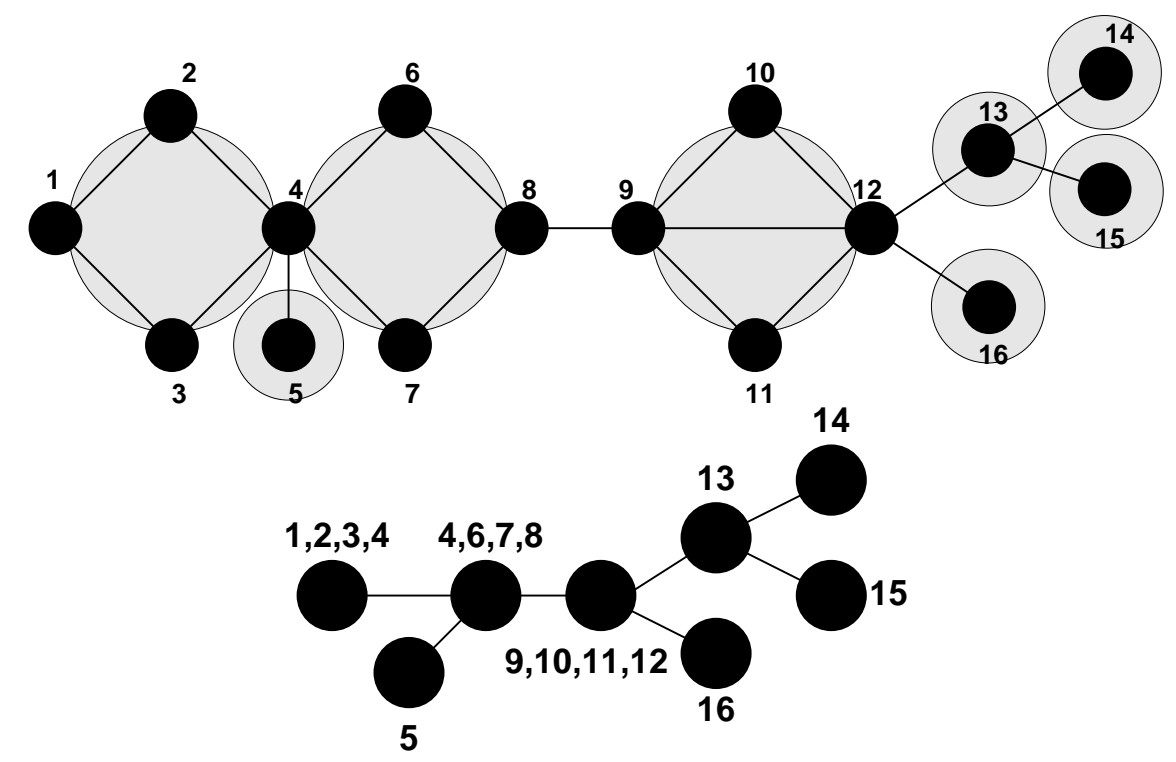

Figure 1: An example graph and one of its associated block graphs.

at least one support in every other variable. Arc-consistent means 2-consistent, path-consistent means 3 -consistent. Strong $k$-consistency is $l$-consistency for all $l \leq k$.

The width of a vertex $v$ w.r.t. a given vertex ordering is the number of adjacent vertices of $v$ preceding it in the ordering. The width of the ordering is the maximum width of all vertices w.r.t. it. The width of a graph is the minimum width of all its orderings. The $j$-width of a vertex $v$ w.r.t an ordering is the minimum, for $k=1$ to $j$, of the width of $k$ consecutive vertices up to and including $v$. The $j$-width of an ordering is the maximum width of all vertices w.r.t. it. The $j$-width of a graph is the minimum widths of all its ordering.

A prefix of an ordering is a non-empty set $S$ of vertices which only consists of a certain vertex $v$ and all its predecessors w.r.t. the ordering. An ordering of a graph is prefix-connected if each graph, induced by a prefix of the ordering, is connected.

The graph in Figure 1 consists of 8 blocks: $\{1,2,3,4\},\{4,6,7,8\},\{5\},\{9,10,11,12\},\{13\}$, $\{14\},\{15\}$ and $\{16\}$. The vertices $4,8,9,12$, and 13 are articulation points. The edges $(4,5)$, $(8,9),(12,13),(12,16),(13,14)$, and $(13,15)$ are bridges. The width of the graph is 2 . The 3 -width of the graph is 1 because 4 is the size of the largest block.

\section{Block-Consistency}

In this section, we define block-consistency ( $\mathrm{BC}$ ). $\mathrm{BC}$ is a weaker kind of consistency, in the sense that it requires restrictions on less tuples. Nevertheless, we will show that strong directed $k$-BC is sufficient for strong directed $k$-consistency if the given ordering is prefix-connected. Furthermore, strong $k$-BC is sufficient for directed consistency w.r.t. each prefix-connected ordering.

First, we define $k$-block-consistency.

Definition 1 A CSP is $k$-block-consistent $(k-B C)$ if each allowed instantiation of $k-1$ variables in the same block has at least one support in every other variable.

Strong $k$-block-consistency is $l$-block-consistency for all $l \leq k$. Directed block-consistency w.r.t. an ordering is block-consistency requiring only supports in variables succeeding the tuple in that ordering. 


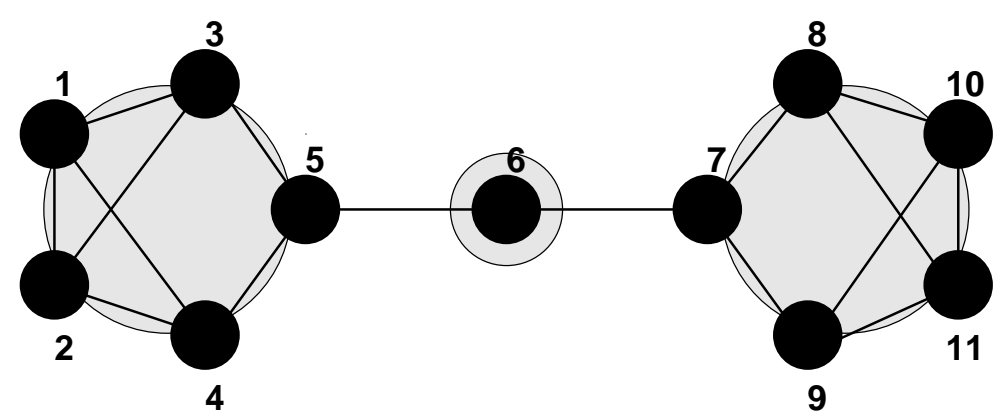

Figure 2: Width-optimal ordering.

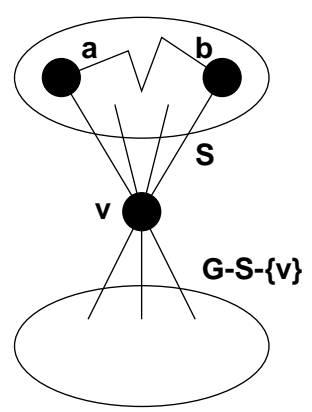

Figure 3:

The adjacency of $v$.

Note that 2-block-consistency is equivalent to arc-consistency, because each vertex obviously is entirely included in one block. Note further that the size of the largest tuple to be considered is bounded by the maximal size of the blocks.

A block of size $k-1$ is trivially $l$-consistent for any $l>k$ since there are no tuples larger than $k-1$. Therefore, strong $k$-block-consistency is enforced as soon as each block of size $l<k$ is strongly $(l+1)$-consistent, and every other block is strongly $k$-consistent.

The enforcement of consistencies higher than arc-consistency has the property that it may add new constraints which possibly increase the width of the graph. The block structure may also be destroyed. Of course, BC enforcement may also insert new edges and increase the width, but the block structure is preserved.

\section{Lemma 1 The block structure is preserved during block-consistency enforcement.}

Proof Block-consistency enforcement results in restrictions for tuples where all vertices are in the same block. Therefore only edges are inserted between vertices which already are biconnected. Thus, no two vertices can become biconnected.

Consequently, all properties defined in terms of blocks are invariant during $\mathrm{BC}$ enforcement. E.g. the width of the graph is bounded to the size of the largest block, the $(b-1)$-width of the graph is always 1 where $b$ is the size of the largest block. Additionally, the set of tuples which must be considered for $\mathrm{BC}$ does not change during $\mathrm{BC}$ enforcement. This is a major advantage compared to classical consistency where the insertion of a edge may destroy the width of the graph, such that the enforcement of the appropriate consistency tends to make the graph complete.

The graph in Figure 2 has width 2. A width-optimal ordering is: $\langle 1,2,3,4,5,11,10,9,8,7,6\rangle$. The vertex 6 is at the end of all width-optimal orderings, because it is the only vertex with degree 2. If, however, the variables are instantiated according to such an ordering, both blocks are solved separately and the partial solutions are not checked until the last vertex. This, however, is not very efficient, because each partial solution is constructed for each other partial solution from scratch. More efficiency may be achieved if vertex 6 is instantiated first, given that the domains of the variables are all of similar size. This approach splits the problem into two sub-problems of smaller size and solves them separately.

The next step shows that the intersection of the adjacency of a vertex with each connected set entirely lies in one block. This property will be used to show the following lemma.

Lemma 2 The intersection of the adjacency of each vertex $v \in V$ and each connected set $S$, $S \subseteq V, v \notin S$, is either empty or belongs entirely to the same block. 
Proof If the adjacency of $v$ in $S$ is empty or contains one vertex, the assumption holds. If it contains two or more vertices, we have at least two paths between any two vertices $a, b \in S$. One path because $S$ is connected and another through $v$, namely $(a, v, b)$. These paths are vertex disjoint and therefore $a$ and $b$ belong to the same block. This holds for any $a, b \in S$ in the adjacency of $v$ and therefore the whole adjacency belongs to the same block (c.f. Figure 3 ).

Now we are ready to state our two main theorems. Both theorems require the use of prefixconnected orderings. This may seem to be a restriction for the choice of variable ordering, but it is mostly desirable to instantiate variables which are adjacent to at least one already instantiated variable. It does not make sense to chose a variable which has no restriction to already instantiated ones.

The first theorem assumes the existence of a prefix-connected ordering which is known in advance and which does not change during search. A weaker consistency has to be computed to achieve directed consistency than in the dynamic case.

Theorem 1 A CSP is strongly directed $k$-consistent w.r.t. a given ordering if the ordering is prefix-connected and the CSP is strongly directed $k$-block-consistent.

Proof To enable directed $k$-consistency, we must show that each $k$-tuple has a support in variables which succeed all vertices in that tuple. Since each prefix of the given ordering is connected, we can use Lemma 2 to show that the intersection of the adjacency of each future variable $v$ and the already instantiated variables entirely lies in one block. Therefore, strong $k$-block-consistency of that block guarantees that there is at least one support for $v$.

Theorem 1 makes algorithms more efficient because of three reasons. First, blocks smaller than $k$ must only be considered up to the induced width of the block limited by the block size. Second, restrictions between variables belonging to more than one block can be skipped. Third, the block structure is preserved and is known in advance.

Theorem 1 holds if the variable ordering is known in advance and fixed during search. However, if we want to allow a dynamical ordering (e.g. for backjumping), more consistency must be applied as shown in the next theorem.

Theorem 2 A CSP is strongly directed $k$-consistent w.r.t. each prefix-connected ordering if it is strongly $k$-block-consistent.

Proof Strong $k$-block-consistency requires that each $l$-tuple, $l<k$, of variables which belong to the same block has a support in each other variable $v$. However, the intersection of the adjacency of each $v$ and the already instantiated variables w.r.t each prefix-connected ordering lies entirely in one block (Lemma 2). This guarantees the needed support for all tuples in all prefix-connected orderings.

Theorem 2 gives us the opportunity to switch the variable ordering during search dynamically, as long as the ordering remains prefix-connected. The advantage must be paid by computing block-consistency instead of directed block-consistency. It is an extension to adaptive consistency and to the solving scheme based on $j$-width, both of which take into account only one given prefix-connected ordering. As shown above, this is not necessarily a good choice. BC, however, provide an explicit way of making use of blocks. Furthermore, it is not a priori clear whether the enforcement of adaptive consistency does preserve the graph structure.

The vertex numbering in Figure 4 determines a variable ordering which is prefix-connected. Making this graph strongly directed path-consistent w.r.t. this ordering is reduced to the application of strong directed 2-block-consistency. This would at most produce restrictions on the 


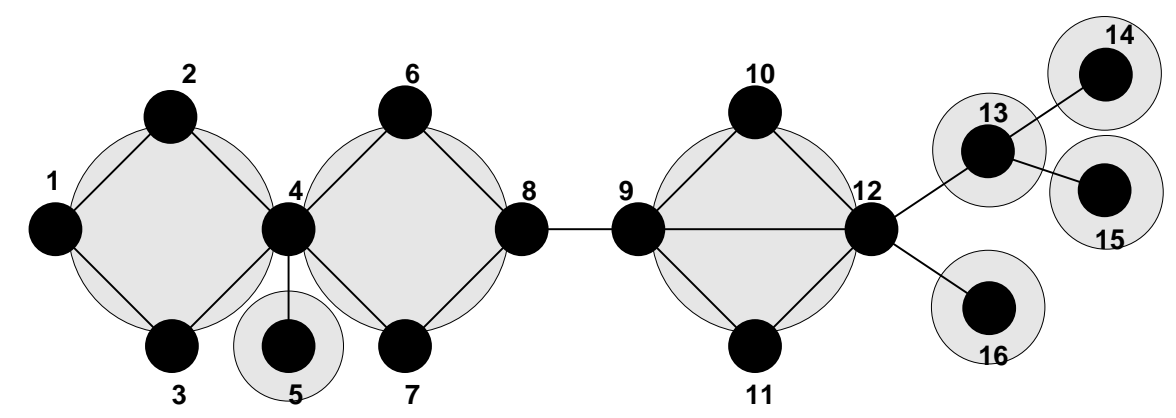

Figure 4: Block-consistency.

(ordered) pairs $(1,4),(2,3),(4,8),(6,7),(10,11)$. All tuples belonging to more than one block (e.g. $(1,10),(2,8))$ do not have to be considered. Furthermore, the blocks built by the vertices $5,13,114,15,16$ need only be made directed arc-consistent. The edges $(4,5),(8,9),(12,13)$, $12,16),(13,14)$, and $(13,15)$ will never be changed because they are bridges.

$\mathrm{BC}$ enforcement is not an isolated solving technique, but can elegantly be added to existing solving algorithms just like directed consistency and adaptive consistency. The necessary measures are:

- The block structure does not change during BC enforcement as shown in Lemma 1. Therefore it only has to be computed once in advance. This can be done in time $O(|V|+$ $|E|)$ as shown by TARJAN in [Tar72] and will not affect the asymptotic bounds of the solving.

- During solving, adapt the following procedure: Whenever consistency for a variable tuple is considered, first check whether the tuple entirely lies in one block. If so, proceed as usual, if no, skip that tuple. The check can be performed in time $O(k)$.

As mentioned before, 2-block consistency is equivalent to 2-consistency. Therefore, solving is only affected if more than arc-consistency is computed. Then, the block structure represents an elegant way of splitting the graph into only slightly interfering subproblems.

\section{Localization}

The concepts of redundancy and inconsistency play an important role in constraint satisfaction. Consistency enforcement for example is a method which adds redundancy to the network, in order to make the constraints more explicit. Forward checking is an algorithm which applies consistency in the variables not yet instantiated, in order to detect inconsistencies as soon as possible. In some applications - e.g. CAD-systems [LM96], [SOMS97]—it is important to inform the user about redundancy, i.e. whether a constraint is (partially) implied by other constraints. Redundancy should be avoided in good CAD-models as it may create problems during the mounting process. In order to eliminate redundancy, the region where it occurs should be encircled as exact as possible.

Redundancy and inconsistency both occur if restrictions on a variable tuple are derived by a direct constraint $C_{i j}$ and by constraints over paths in the remaining graph. Redundancy means that the entire restriction of the constraint are already induced by the remaining graph, inconsistency means that the solution sets are disjoint. Redundancy implies that the constraint could be removed from the problem and the solution set would remain the same. Often, however, a constraint is not totally redundant, but only some value pairs are restricted more than once. 

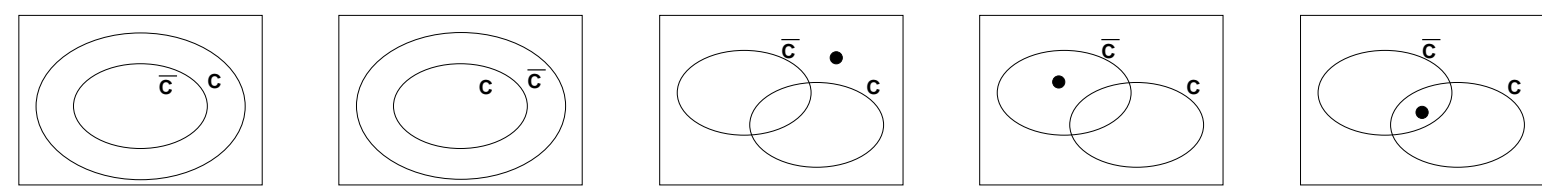

Figure 5: Redundant, explicit, partially redundant, partially explicit, and satisfiable constraint.

This indicates that the constraint could be loosened a little without changing the solution set. We will call such a constraint partially redundant.

The arguments over the existence of paths in the remaining network indicates that redundancy and inconsistency must have a connection with biconnected components. To show this we first give a little more formal definition of the terminology. These definitions are mostly adapted from [DD87]. The network-induced constraint $\bar{C}_{i j}$ between two variables $i$ and $j$ is the set of all value pairs which are allowed by the graph except the (direct) constraint between $i$ and $j$. A constraint $C_{i j}$ is redundant if $C_{i j} \supseteq \bar{C}_{i j}$. A constraint $C_{i j}$ is partially redundant if there exists at least one value tuple of $D_{i} \times D_{j}$ which is neither in $\bar{C}_{i j}$ nor in $C_{i j}$. A constraint $C_{i j}$ is explicit if $C_{i j} \subseteq \bar{C}_{i j}$. A constraint $C_{i j}$ is partially explicit if there exists at least one value tuple of $D_{i} \times D_{j}$ which is not $C_{i j}$ but in $\bar{C}_{i j}$. A constraint $C_{i j}$ is satisfiable if $C_{i j} \cap \bar{C}_{i j} \neq \emptyset$. We also say that a constraint which is not satisfiable is inconsistent.

The Venn diagrams for the different kind of constraints are shown in Figure 5. The dots indicate in which region a value tuple must exist. If a constraint $C_{i j}$ is redundant, then it is also partially redundant. In fact, each value tuple for $i$ and $j$ which is not in $C_{i j}$ and also not in $\bar{C}_{i j}$. If a constraint is explicit then it is also partially explicit. Analogous, each tuple for $i$ and $j$ which is in $\bar{C}_{i j}$ is not in $C_{i j}$. We can see that if a constraint is partially explicit and not totally explicit then it is either partially redundant or inconsistent. Redundancy, explicitness and satisfiability of a constraint $C_{i j}$ are related to the fact whether $i$ and $j$ belong to the same block.

Theorem 3 If a constraint $C_{i j}$ is (partially) redundant, then $i$ and $j$ belong to the same block.

Proof We show that a constraint $C_{i j}$ can never be partially redundant if $i$ and $j$ do not belong to the same block. If $i$ and $j$ do not belong to the same block, then $\bar{C}_{i j}$ equals $D_{i} \times D_{j}$. A necessary condition for (partial) redundancy, however, is the existence of a value tuple which is neither in $C_{i j}$ nor in $\bar{C}_{i j}$. However, there is no tuple which is not in $\bar{C}_{i j}$ and therefore $C_{i j}$ cannot be (partially) redundant.

It directly follows that a constraint $C_{i j}$ is always explicit if $i$ and $j$ do not belong to the same block. Bridges connect vertices of different blocks and they are therefore always explicit and never redundant.

Theorem 4 If a constraint $C_{i j}$ is not satisfiable, then $i$ and $j$ belong to the same block.

Proof We show that a constraint can never be inconsistent if $i$ and $j$ do not belong to the same block. If $i$ and $j$ do not belong to the same block, then $\bar{C}_{i j}=D_{i} \times D_{j}$. Each non-empty constraint, however, is a proper subset of $D_{i} \times D_{j}$ and therefore the intersection with $\bar{C}_{i j}$ is not empty and, hence, $C_{i j}$ is satisfiable.

As we have seen, redundancy and inconsistency can only be observed in constraints between variables of the same block. This gives us the opportunity to encircle the source of these properties down to the block to which the constrained variables belong. In addition, a constraint between variables which are not in the same block is always explicit. Consistency enforcement 
aims in making each constraint as explicit as possible [DD87]. Bridges are always explicit. This is an additional argument, why $\mathrm{BC}$ enforcement need not to treat bridges.

Consistency enforcement adds additional redundancy to the graph, in order to make the constraints as explicit as possible. However, if $k$-consistency, $k>1$, is enforced, the block structure may be destroyed due to newly added edges and redundancy and inconsistency are no longer confined to the blocks. BC leaves the block structure intact and does not add paths if there have not yet been at least two. Therefore, the location of redundancy and inconsistency remain invariant during $\mathrm{BC}$ enforcement, which is very important for practical applications.

\section{$5 \quad$ Blocks and Width}

In this section, we analyze how the width of a graph is bounded to its block structure. FreUDER has shown in [Fre85] that the $(b-1)$-width of a graph is 1 where $b$ is the size of the largest block. This result can be achieved by traversing the block graph and enumerating the vertices in prefix order. But he does not indicate how the width of a graph is connected to any block parameters.

First, we observe that the width of the entire graph is at least as high as the maximum width of all subgraphs and therefore at least as high as the width of all blocks. This is not an exact bound as can be seen in Figure 6 . The graph consists of two blocks: $\{1,2,3,4,5\}$ and $\{5,6,7,8,9\}$. Both block induced subgraphs have a width of 2 . In the entire graph, however, each vertex has degree 3 or higher which is a lower bound for the width. There are indeed orderings with width 3 , one indicated by the numbers of the vertices. The reason for the increased width are the articulation points, which lie in more than one block and therefore have a larger degree in the entire graph than in the block-induced subgraphs.

However, the upper bound of the width of a graph is not unrelated to the width of the blocks. The orderings achieving minimal width for each block can put together to form an ordering for the entire graph. The only problem is that we need to rearrange the articulation points. But as shown in the following lemma, this increases the width of the ordering by at most 1 .

Lemma 3 Moving one vertex in front within a given ordering increases the width of this ordering by at most 1 .

Proof If a vertex $v$ is moved in front, then its width w.r.t. the ordering is not increased, because no additional edge will be counted. The width of each vertex $w \in \operatorname{adj}(v), w<v$, however, is increased by 1 when $v$ passes $w$. This is the only change affecting the vertex $w$. Therefore the width of each vertex is increased by at most one. The width of the ordering is also increased by at most 1 .

An example for a reordering is given in Figure 7. The ordering has width 2. After vertex 4 is moved to front, all adjacent vertices which are passed have increased width. Vertex 2 has width 2 instead of 1 , vertex 3 has width 3 instead of 2 . The vertex 4 itself has width 0 . The width of the new ordering is increased to 3 . Note that it is possible that the width of an ordering remains the same or even becomes smaller although a vertex is moved to front.

This property of reorderings can be used to construct an ordering for the whole graph out of orderings for blocks. Additionally, it can be shown by example that both bounds are tight.

Theorem 5 The width $k$ of a graph $G$ is bounded by $k_{\max } \leq k \leq k_{\max }+1$ where $k_{\max }$ is the maximal width of the block induced subgraphs of $G$. Both bounds are tight. 


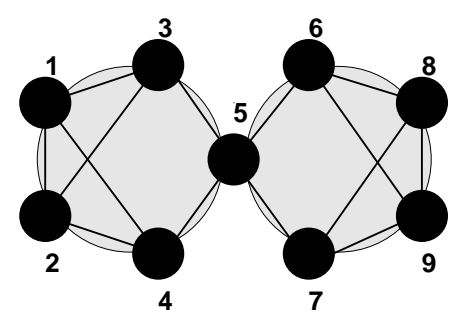

Figure 6: A graph with $k=k_{\max }+1$.

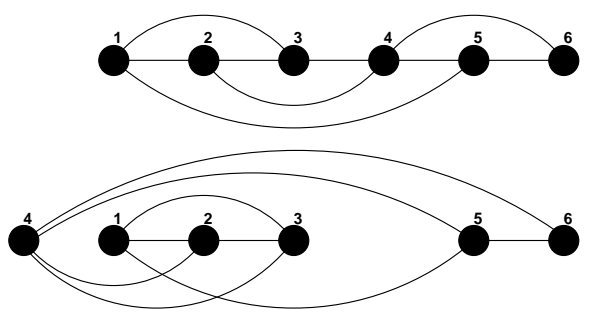

Figure 7: Reordering.

Proof A width-optimal ordering of the entire graph can be used to derive an ordering for a block induced subgraph by removing all vertices which are not in that block. Removal of vertices may only decrease the width of that ordering. Therefore the width of the ordering for the block is at most $k$. This proves that $k_{\max } \leq k$.

We construct an ordering with width $k_{\max }+1$ in the following way. Since the block graph is a forest, there must be at least one block which has at most one articulation point. We take the vertices except the articulation point in the width-optimal ordering for that block to be the end of the global ordering. Then we remove the block from the block graph. We take the next block which has at most one articulation point in the remaining block graph and put its ordering except the articulation point in front of the ordering of the last block. We proceed until the block graph is empty. Articulation points of the starting graph are collected as soon as they are no more articulation points in the temporary graph. This gives us a global ordering where the width of the vertices is the same as in the block orderings with the only difference that one articulation point might be moved to front. As we have seen in Lemma 3 may increase the width of each vertex of that block by at most one. Therefore the constructed ordering has a width which is at most $k_{\max }+1$. Both bounds are tight. The lower bound is obviously reached by a graph with only one block. An example where the upper bounds is reached is shown in Figure 6.

In Figure 8, width-optimal orderings for the block induced subgraphs are $\langle 1,2,3,4,12\rangle$, $<8,7,6,5,12\rangle$ and $\langle 9,10,11,12\rangle$. The maximal width of the blocks $k_{\max }$ is 2 . The block $9,10,11,12$ has only one articulation point (12). We remove all vertices of this block except its articulation point and put them at the end of the global ordering. Then we remove the vertices $1,2,3,4$ of the next block and finally the remaining vertices of the third block with vertex 12 at its normal place as it is no more an articulation point. This gives us the global ordering $<8,7,6,5,12,1,2,3,4,9,10,11>$. We see that the vertices $3,4,9$, and 11 have an increased width due to the fact that vertex 12 has been move to front of them. The width of the global ordering is 3 which is $k_{\max }+1$. Note that this ordering must not be the optimal one as can be seen by the fact that by switching 11 and 10 at the end. The construction only serves to show the existence of an ordering with a width at most $k_{\max }+1$.

An upper bound for the width of a block $B$ is $|B|-1$. In such a case, however, each ordering is width-optimal and we do not need to rearrange the articulation points. Therefore the width of a graph is also bounded by the size of the largest block as shown in the following lemma.

Theorem 6 If the size $b$ of the largest block is $\geq 2$ then the width $k$ of a graph $G$ is $\leq b-1$.

Proof We observe that the only graphs of size $n$ which have a width of $n-1$ are either complete or have exactly one vertex. For such graphs, however, each ordering is width-optimal. If a block $B$ has a width of $|B|-1$, the articulation points are not be moved to front in the ordering constructed in Theorem 5. Therefore the width of the global ordering is bounded by $b-1$ where $b$ is the maximum of 2 and the size of the largest block. 


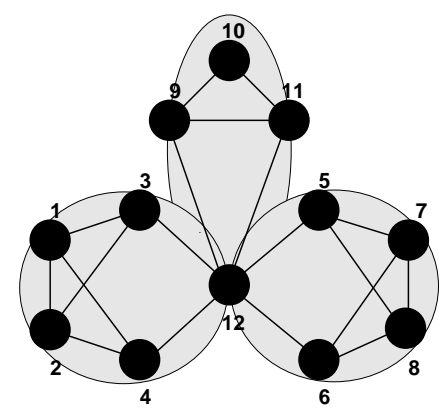

Figure 8: Construction of a width-optimal ordering.

A graph is a forest, if the size of the largest block is 1 . The width $k$ of a forest is $\leq 1$. Generally, a CSP can be solved backtrack-free if it is strongly $k+1$-consistent where $k$ is the width of the graph [Fre82]. In addition, in [Fre85] there was shown that a CSP can also be solved in time exponential in the size of the largest block. This approach is based on the concept of $j$-width. He gives an ordering which achieves a $(b-1)$-width of 1 where $b$ is the size of the largest block. We derive the same result without using the (implicit) concept of $j$-width. We show that strong directed $b$-BC is sufficient to allow backtrack-free search where $b$ is the maximum of 2 and the largest block size.

Theorem 7 There exists a backtrack-free vertical search order if the graph is strongly directed $b$-block consistent w.r.t the ordering where $b$ is the maximum of 2 and the size of the largest block.

Proof In Theorem 1 we have shown that strongly directed $k$-BC is sufficient for strongly $k$-consistency w.r.t a prefix-connected ordering. The width-optimal ordering in Theorem 5 is prefix-connected. Directed $k$-consistency is sufficient for $k$-consistency w.r.t. a given ordering. Strong $k$-consistency is sufficient for backtrack-free search if the width of the graph is $k-1$. The width of a graph is bounded by $b-1$ where $b$ is the maximum of 2 and the size of the largest block. Therefore strongly directed $b$-BC is sufficient for global consistency and there is a backtrack-free vertical search order, namely the one shown in [Fre85].

Assume that the graph in Figure 4 is strongly directed 3-block-consistent w.r.t the ordering indicated by the numbering. First we assign vertex 1 , vertex 2 can be instantiated because BC implies arc-consistency of 1 . Vertex 3 equally. Vertex 4 can be assigned because 2 and 3 are path-consistent. Vertex 5 can be instantiated because 4 is arc-consistent and so on. As soon as we come to vertex 13 , which is an articulation point, arc-consistency is needed to instantiate vertex 14 . The arc-consistency is not implied by the strong directed $k_{B}$-block-consistency, because $k_{B}$ is 1 in this case.

Strong directed $k$-BC can be computed in time exponential in $W^{*}$ where $W^{*}$ is the largest induced width of all block which may be smaller than $b-1$. Additional, BC provides a more explicit way of analyzing the running time a priori, since the block structure is preserved during enforcement.

\section{Conclusions}

Blocks are a structure which has an impact on CSPs in several ways. First, they allow us to define block consistency which is a weaker, but nevertheless sufficient kind of consistency if prefix-connected orderings are used. Such orderings, however, are a natural choice and no 
really a restriction. Moreover, BC enforcement preserves the block structure such that each block-based observation remains invariant during $\mathrm{BC}$ enforcement. Second, redundancy and inconsistency can be detected on the block level. We have shown that a constraint may be inconsistent or redundant only if the constrained variables belong to the same block. Third, the width of the graph is bounded by the width of the blocks.

Further research includes testing BC enforcement empirically. Doing so, it is not useful to take random problems, since they consist of only one block even if they are sparse. However, many practical problems (e.g. the assembly problem in a CAD-system) typically consist of several or even a lot of blocks because modeling humans try to split the whole problem into loosely connected subproblems. This is reflected in strategies as "divide and conquer" and hierarchical approaches like "bottom-up" and "top-down". In our opinion, it will be very interesting to exploit this assumption about constraint problems, e.g. by using the concept of blocks.

\section{Acknowledgments}

We would like to thank H. Meyer auf' $m$ Hofe and A. Below for reading an earlier version of this paper. We would also like to thank Th. Raschle and N. Galli for many fruitful comments on graph-related topics.

\section{References}

[DD87] A. Dechter and R. Dechter. Removing Redundancies in Constraint Networks. In Proceedings of the National Conference on Artificial Intelligence, pages 105-109. AAAI Press, 1987.

[DP88] Rina Dechter and Judea Pearl. Network-Based Heuristics For Constraint-Satisfaction Problems. In Laveen Kanal and Vipin Kumar, editors, Search in Artificial Intelligence. Springer-Verlag, New York, 1988.

[Fre82] Eugene C. Freuder. A Sufficient Condition for Backtrack-Free Search. Journal of the ACM, 29(1):2432,1982 .

[Fre85] Eugene C. Freuder. A sufficient condition for backtrack-bounded search. Journal of the ACM, $32(4): 755-761,1985$.

[LM96] R. S. Latham and A. E. Middleditch. Connectivity analysis: a tool for processing geometric constraints. Computer-Aided Design, 28(11):917-928, 1996.

[SOMS97] B. Seybold, G. Ogan, F. Metzger, and K. Simon. Interactive aspects of constraint-based assembly modeling. In CP97 Workshop on The Theory and Practice of Dynamic Constraint Satisfaction, November 1997.

[Tar72] Robert Tarjan. Depth-first search and linear graph algorithms. SIAM Journal on Computing, 1(2):146-160, June 1972. 